\title{
DISPERSION ESTIMATES FOR DISCRETE SCHRÖDINGER AND KLEIN-GORDON EQUATIONS
}

\author{
E. A. KOPYLOVA
}

Abstract. The long-time asymptotics is derived for solutions of the discrete 3dimensional Schrödinger and Klein-Gordon equations.

\section{$\S 1$. Introduction}

We consider the 3-dimensional discrete version of the Schrödinger equation,

$$
\left\{\begin{array}{l}
i \dot{\psi}(x, t)=H \psi(x, t):=(-\Delta+V(x)) \psi(x, t), \quad \mid x \in \mathbb{Z}^{3}, \quad t \in \mathbb{R}, \\
\left.\psi\right|_{t=0}=\psi_{0},
\end{array}\right.
$$

where $\Delta$ stands for the difference Laplacian in $\mathbb{Z}^{3}$, defined by

$$
\Delta \psi(x)=\sum_{|y-x|=1} \psi(y)-6 \psi(x), \quad x \in \mathbb{Z}^{3}, \quad \psi: \mathbb{Z}^{3} \rightarrow \mathbb{C} .
$$

We denote by $\mathcal{V}$ the set of real-valued finitely supported functions on the lattice $\mathbb{Z}^{3}$. Concerning the potential $V$, we assume that $V \in \mathcal{V}$. Then for any $\psi_{0} \in l^{2}=l^{2}\left(\mathbb{Z}^{3}\right)$ there exists a unique solution $\psi(x, t) \in C\left(\mathbb{R}, l^{2}\right)$ of the Cauchy problem (1.1), and the charge conservation law $\|\psi(\cdot, t)\|_{l^{2}}=$ const is fulfilled.

It is well known that, with the help of the Fourier-Laplace transformation relative to the variable $t$, the properties of the nonstationary equation can be deduced from the properties of the resolvent $R(\omega)=(H-\omega)^{-1}$ of the Schrödinger operator $H$.

We are going to use the weighted Hilbert spaces $l_{\sigma}^{2}=l_{\sigma}^{2}\left(\mathbb{Z}^{3}\right)$ with the norms

$$
\|u\|_{l_{\sigma}^{2}}=\left\|\left(1+x^{2}\right)^{\sigma / 2} u\right\|_{l^{2}}, \quad \sigma \in \mathbb{R} .
$$

Let

$$
B\left(\sigma, \sigma^{\prime}\right)=\mathcal{L}\left(l_{\sigma}^{2}, l_{\sigma^{\prime}}^{2}\right)
$$

denote the space of bounded linear operators from $l_{\sigma}^{2}$ to $l_{\sigma^{\prime}}^{2}$.

The spectrum of the operator $H$ consists of the continuous spectrum and real eigenvalues $\mu_{j}, j=1, \ldots, n$. Note that $n \leq N$, where $N$ is the number of points in the support of $V$ (see [4, Chapter I, Theorem $\left.13^{b i s}\right]$ ).

The continuous spectrum of $H$ coincides with the interval [0,12], which is the range of the symbol $\phi(\theta)=4\left(\sin ^{2} \frac{\theta_{1}}{2}+\sin ^{2} \frac{\theta_{2}}{2}+\sin ^{2} \frac{\theta_{3}}{2}\right)$ of the difference Laplace operator $H_{0}=-\Delta$.

We pay special attention to the points $\omega_{k}=4 k \in[0,12], k=0,1,2,3$, which are critical values of the symbol, i.e., the values of the symbol at the critical points.

2010 Mathematics Subject Classification. Primary 39A14, 35L10.

Key words and phrases. Discrete Schrödinger and Klein-Gordon equations, lattice, Cauchy problem, long-time asymptotics, weighted norms.

Supported partly by FWF (grant no. P19138-N13), DFG (grant no. 436 RUS 113/929/0-1), and RFBR (grants nos. 07-01-00018a and 06-01-00096). 
Our main results are as follows. For "a generic potential" $V \in \mathcal{V}$ (see Definition 3.3), we obtain:

a) the existence of the limits $R(\omega \pm i 0)$ ("limiting absorption principle") on the continuous spectrum in the norm of $B(\sigma ;-\sigma)$ with $\sigma>3 / 2$;

b) the Puiseux expansion for the resolvent at the singular spectral points $\omega_{k}$ :

$$
R\left(\omega_{k}+\omega\right)=D_{k}+\mathcal{O}(\sqrt{\omega}), \quad \omega \rightarrow 0,
$$

in the norm of $B(\sigma ;-\sigma)$ with $\sigma>7 / 2$.

Then, for initial data $\psi_{0} \in l_{\sigma}^{2}$ with $\sigma>11 / 2$ we deduce the following long-time asymptotics:

$$
\left\|e^{-i t H}-\sum_{j=1}^{n} e^{-i t \mu_{j}} P_{j}\right\|_{\mathcal{B}(\sigma,-\sigma)}=\mathcal{O}\left(t^{-3 / 2}\right), \quad t \rightarrow \infty .
$$

Here the $P_{j}$ are the orthogonal projections in $l^{2}$ onto the eigenspaces of $H$ corresponding to the discrete eigenvalues $\mu_{j}$.

We also obtain similar results for the discrete Klein-Gordon equation

$$
\left\{\begin{array}{l}
\ddot{\psi}(x, t)=\left(\Delta-m^{2}-V(x)\right) \psi(x, t), \quad \mid x \in \mathbb{Z}^{3}, \quad t \in \mathbb{R} . \\
\left.\psi\right|_{t=0}=\psi_{0},\left.\dot{\psi}\right|_{t=0}=\pi_{0},
\end{array}\right.
$$

Some comments on previous results in this direction are in order. For the first time, the difference Schrödinger equation was considered by Èskina in [14. She proved the limiting absorption principle for the matrix entries of the resolvent. The asymptotic expansion of the matrix entries of the resolvent $R(\omega)$ at the critical points $\omega_{k}$ was obtained by Islami and Vainberg [5] in the 2-dimensional case. They used this expansion to prove the long-time asymptotics for the solutions of the Cauchy problem for the difference wave equation. The present paper differs from [5] mainly by the fact that here all asymptotic expansions are valid in the weighted function spaces $l_{\sigma}^{2}$ rather than on compact sets. Such expansions may be useful for the study of nonlinear evolutionary equations.

The asymptotic expansion of the resolvent and the long-time asymptotics (1.3) for hyperbolic PDEs in $\mathbb{R}^{n}$ (continuous case) were obtained earlier in [10, 2, 3, and for the Schrödinger equation in [6, 7, 11, see also 12 for an up-to-date review. We use the main ideas of those papers.

The results of the present paper extend the results of 8 and 9 from 1- and 2dimensional difference equations to 3 -dimensional ones. In the 3-dimensional case, we encounter more difficult analytic problems, because of the diversity of the types of critical points.

The paper is organized as follows. In $\$ 2$ we prove the limiting absorption principle and derive the Puiseux asymptotic of the free resolvent. In $\$ 3$ we extend these results to the case of a perturbed resolvent. In $\$ 4$ we prove the long-time asymptotics (1.3). In $\$ 5$ we consider the discrete Klein-Gordon equation. In Appendix $\mathrm{C}$ we apply our results to construct asymptotic scattering states.

\section{§2. Free Resolvent}

We start with the investigation of the unperturbed problem for equation (1.1) with $V(x)=0$. The discrete Fourier transform of $u(x) \in l^{2}\left(\mathbb{Z}^{3}\right)$ is defined by the formula

$$
\widehat{u}(\theta)=\sum_{x \in \mathbb{Z}^{3}} u(x) e^{i \theta x}, \quad \theta \in T^{3}:=\mathbb{R}^{3} / 2 \pi \mathbb{Z}^{3} .
$$


After the Fourier transformation, the operator $H_{0}=-\Delta$ becomes the operator of multiplication by $\phi(\theta):=6-2 \sum_{j=1}^{3} \cos \theta_{j}=4 \sum_{j=1}^{3} \sin ^{2} \frac{\theta_{j}}{2}$ :

$$
-\widehat{\Delta u}(\theta)=\phi(\theta) \widehat{u}(\theta), \quad \theta \in T^{3} .
$$

Thus, the spectrum of $H_{0}$ coincides with the range of the function $\phi$, i.e., Spec $H_{0}=$ $\Sigma:=[0,12]$. We denote by $R_{0}(\omega)=\left(H_{0}-\omega\right)^{-1}$ the resolvent of the difference Laplacian. Then the kernel of the resolvent $R_{0}(\omega)$ looks like this:

$$
R_{0}(\omega, x-y)=\frac{1}{8 \pi^{3}} \int_{T^{3}} \frac{e^{-i \theta(x-y)}}{\phi(\theta)-\omega} d \theta, \quad \omega \in \mathbb{C} \backslash \Sigma .
$$

Lemma 2.1. The free resolvent $R_{0}(\omega)$ is an analytic function of $\omega \in \mathbb{C} \backslash \Sigma$ with values in $\mathcal{B}\left(\sigma, \sigma^{\prime}\right)$ for any $\sigma, \sigma^{\prime} \in \mathbb{R}$.

Proof. For a fixed $\omega \in \mathbb{C} \backslash \Sigma$, we have $\phi(\theta)-\omega \neq 0$ for $\theta \in T^{3}$. Therefore, $\phi(\theta+i \xi)-\omega \neq 0$ for $\theta \in T^{3}$ and $\xi \in \mathbb{R}^{3}$ provided $\xi \neq 0$ is sufficiently small. Hence, the function $1 /(\phi(\theta)-\omega)$ admits analytic continuation to a complex neighborhood of the torus $\left\{\theta+i \xi: \theta \in T^{3}, \xi \in \mathbb{R}^{3}:|\xi|<\delta(\omega)\right\}$ with $\delta(\omega)>0$. Therefore, the Paley-Wiener arguments show that

$$
\left|R_{0}(\omega, x-y)\right| \leq C(\delta) e^{-\delta|x-y|}
$$

for any $\delta<\delta(\omega)$. Hence, $R_{0}(\omega) \in \mathcal{B}\left(\sigma, \sigma^{\prime}\right)$ by the Schur lemma.

2.1. Limiting absorption principle. Now we are interested in the traces of the analytic function $R_{0}(\omega)$ at the cut $\Sigma$. Consider

$$
R_{0}(\omega \pm i \varepsilon, z)=\frac{1}{8 \pi^{3}} \int_{T^{3}} \frac{e^{-i \theta z}}{\phi(\theta)-\omega \mp i \varepsilon} d \theta, \quad z \in \mathbb{Z}^{3}, \quad \omega \in \Sigma, \quad \varepsilon>0 .
$$

Note that the limiting distribution $\frac{1}{\phi(\theta)-\omega \mp i 0}$ is well defined if $\omega$ is not a critical value of the function $\phi(\theta)$, i.e., $\omega \neq 0,4,8,12$. The following limiting absorption principle is valid.

Proposition 2.2. For $\sigma>3 / 2+k$, the following limits exist as $\varepsilon \rightarrow 0+$ :

$$
\partial_{\omega}^{k} R_{0}(\omega \pm i \varepsilon) \stackrel{\mathcal{B}(\sigma,-\sigma)}{\longrightarrow} \partial_{\omega}^{k} R_{0}(\omega \pm i 0), \quad \omega \in \Sigma \backslash\{0,4,8,12\} .
$$

Proof. i) Let $k=0$. First, we follow [14 to prove (2.4) for any fixed $z$. Let $\chi_{j}(\theta)$, $j=1, \ldots, l$, be a sufficiently fine partition of unity on the torus $T^{3}$, which will be specified below. Then

$$
R_{0}(\omega \pm i \varepsilon, z)=\sum_{j=1}^{l} \frac{1}{8 \pi^{3}} \int_{D_{j}} \frac{\chi_{j}(\theta) e^{-i \theta z}}{\phi(\theta)-\omega \mp i \varepsilon} d \theta=\sum_{j=1}^{l} P_{j}(\omega \pm i \varepsilon, z),
$$

where $D_{j}$ is the support of the function $\chi_{j}$. If $\{\phi(\theta)=\omega\} \cap D_{j}=\varnothing$, then the function $P_{j}(\omega \pm i \varepsilon, z)$ is continuous for $\varepsilon \geq 0$ and

$$
\left|P_{j}(\omega \pm i \varepsilon, z)\right|<C_{j}<\infty, \quad z \in Z^{3}, \quad \varepsilon \geq 0 .
$$

Now, let $S_{j}=\{\phi(\theta)=\omega\} \cap D_{j}$. Then any $\theta \in D_{j}$ can be represented uniquely as $\theta=s+\operatorname{tn}(s)$, where $s \in S_{j}$, and $n(s)$ is the outward unit normal to $S_{j}$ at the point $s$. We introduce the new variables $(s, t)$. Then

$$
P_{j}(\omega \pm i \varepsilon, z)=\frac{1}{8 \pi^{3}} \int_{S_{j}} e^{-i s z} d s \int_{-a(s)}^{b(s)} \frac{\chi_{j}(s+\operatorname{tn}(s)) e^{-i t n(s) z} J(s, t)}{t \psi(s+\operatorname{tn}(s)) \mp i \varepsilon} d t,
$$

where $J(s, t)$ is the Jacobian, $\psi$ is a smooth function, and $a(s), b(s)>0$. Observe that $\left.J(s, t)\right|_{t=0}=1$ and $\left.\psi(s+\operatorname{tn}(s))\right|_{t=0}=|\nabla \phi(s)| \neq 0$, because $\omega \in \Sigma \backslash\{0,4,8,12\}$ is not a critical value of $\phi(\theta)$. We need the following statement. 
Lemma 2.3. Let $\varphi(t, z)$ be a smooth function satisfying

$$
|\varphi(t, z)| \leq C, \quad\left|\partial_{t} \varphi(t, z)\right| \leq C|z|, \quad t \in[-\delta, \delta], \quad z \in \mathbb{Z}^{3},
$$

and let $\psi(t)$ be a smooth function such that $\psi(t) \neq 0$ if $t \in[-\delta, \delta]$. Consider

$$
F( \pm \varepsilon, z)=\int_{-\delta}^{\delta} \frac{\varphi(t, z)}{t \psi(t) \mp i \varepsilon} d t .
$$

Then $F( \pm \varepsilon, z) \rightarrow F( \pm 0, z)$ as $\varepsilon \rightarrow 0+$ for all $z \in \mathbb{Z}^{3}$, and

$$
\sup _{\varepsilon \in(0,1]}|F( \pm \varepsilon, z)| \leq C(\log (1+|z|)+1), \quad z \in \mathbb{Z}^{3} .
$$

Proof. We rewrite $F( \pm \varepsilon, z)$ as

$$
\begin{aligned}
F( \pm \varepsilon, z)= & \varphi(0, z) \int_{-\delta}^{\delta} \frac{d t}{t \psi(0) \mp i \varepsilon}-\varphi(0, z) \int_{-\delta}^{\delta} \frac{(\psi(t)-\psi(0)) t d t}{(t \psi(t) \mp i \varepsilon)(t \psi(0) \mp i \varepsilon)} \\
& +\int_{-\delta}^{\delta} \frac{\varphi(t, z)-\varphi(0, z)}{t \psi(t) \mp i \varepsilon} d t .
\end{aligned}
$$

Then

$$
\begin{aligned}
F( \pm \varepsilon, z) \rightarrow F( \pm 0, z)= & \pm i \pi \frac{\varphi(0, z)}{\psi(0)}-\frac{\varphi(0, z)}{\psi(0)} \int_{-\delta}^{\delta} \frac{\psi(t)-\psi(0)}{t \psi(t)} d t \\
& +\int_{-\delta}^{\delta} \frac{\varphi(t, z)-\varphi(0, z)}{t \psi(t)} d t
\end{aligned}
$$

as $\varepsilon \rightarrow 0+$. By (2.8), the first and second summands on the right in (2.9) can be estimated by a constant independent of $|z| \in \mathbb{Z}^{3}$. We estimate the third term on the right-hand side of (2.9). For $|z|<1 / \delta$, this term can also be estimated by a constant. For $|z|>1 / \delta$, we use (2.8) to show that

$$
\begin{aligned}
\int_{-\delta}^{\delta}\left|\frac{\varphi(t, z)-\varphi(0, z)}{t \psi(t)}\right| d t & =\int_{|t|<1 /|z|} \cdots+\int_{1 /|z|<|t|<\delta} \cdots \\
& \leq \frac{1}{|z|} C|z|+C \log |z| \leq C \log |z| .
\end{aligned}
$$

The lemma is proved.

Lemma 2.3 implies that for all $z \in \mathbb{Z}^{3}, P_{j}(\omega \pm i \varepsilon, z) \rightarrow P_{j}(\omega \pm i 0, z)$ as $\varepsilon \rightarrow 0+$, and

$$
\sup _{\varepsilon \in(0,1]}\left|P_{j}(\omega \pm i \varepsilon, z)\right| \leq C_{j}(\log (1+|z|)+1) .
$$

Obviously, the entire resolvent $R_{0}$ has similar properties. Hence, by the Lebesgue dominated convergence theorem,

$$
\sum_{x, y \in \mathbb{Z}^{3}}\left(1+|x|^{2}\right)^{-\sigma}\left|R_{0}(\omega \pm i \varepsilon, x-y)-R_{0}(\omega \pm i 0, x-y)\right|^{2}\left(1+|y|^{2}\right)^{-\sigma} \rightarrow 0
$$

as $\varepsilon \rightarrow 0+$, with $\sigma>3 / 2$. Then the Hilbert-Schmidt norm of the difference $R_{0}(\omega \pm i \varepsilon)-$ $R_{0}(\omega \pm i 0)$ converges to zero. This proves Proposition 2.2 in the case where $k=0$. 
ii) For $k \neq 0$ we use integration by parts. For instance, let $k=1$. Since $\nabla \phi(\theta) \neq 0$ for $\theta \in D_{j}$, there exists $i \in\{1,2,3\}$ such that $\partial_{i} \phi(\theta) \neq 0$ for $\theta \in D_{j}$. Consequently,

$$
\begin{aligned}
P_{j}^{\prime}(\omega \pm i \varepsilon, z) & =\frac{1}{8 \pi^{3}} \int_{D_{j}} \frac{\chi_{j}(\theta) e^{-i \theta z}}{(\phi(\theta)-\omega \mp i \varepsilon)^{2}} d \theta \\
& =-\frac{1}{8 \pi^{3}} \int_{D_{j}} \partial_{i}\left(\frac{1}{\phi(\theta)-\omega \mp i \varepsilon}\right) \frac{\chi_{j}(\theta) e^{-i \theta z} d \theta}{\partial_{i} \phi(\theta)} \\
& =\frac{1}{8 \pi^{3}} \int_{D_{j}} \frac{1}{\phi(\theta)-\omega \mp i \varepsilon} \partial_{i}\left(\frac{\chi_{j}(\theta) e^{-i \theta z}}{\partial_{i} \phi(\theta)}\right) d \theta .
\end{aligned}
$$

The rest of the proof is similar to the case of $k=0$. Differentiation of the exponential yields an additional factor $z_{i}$, and then the value of $\sigma$ increases by one.

2.2. Asymptotics near critical points. In the sequel, we shall need information on the behavior of the resolvent $R_{0}(\omega)$ near the critical points $\omega_{k}$. We consider the "elliptic" points $\omega_{1}=0, \omega_{4}=12$ and the "hyperbolic" points $\omega_{2}=4, \omega_{3}=8$ separately.

2.2.1. Elliptic points. Here we construct the Puiseux expansion of the free resolvent $R_{0}(\omega)$ near the point $\omega_{1}=0$ (the expansion near the point $\omega_{4}=12$ can be constructed similarly).

Proposition 2.4. Let $N=0,1,2, \ldots$, and let $\sigma>N+3 / 2$. Then the following expansion is valid in $\mathcal{B}(\sigma,-\sigma)$ :

$$
R_{0}(\omega)=\sum_{k=0}^{N} A_{k} \omega^{k / 2}+\mathcal{O}\left(\omega^{(N+1) / 2}\right), \quad|\omega| \rightarrow 0, \quad \arg \omega \in(0,2 \pi) .
$$

Here $A_{k} \in \mathcal{B}(\sigma,-\sigma)$ with $\sigma>k+1 / 2$.

Proof. The resolvent $R_{0}(\omega \pm i 0)$ is represented by the integral (2.2). Fix $0<\delta<1$ and consider $0<|\omega|<\delta^{2} / 2$. We identify $T^{3}$ with the cube $[-\pi, \pi]^{3}$ and represent $R_{0}(\omega, z)$, $z=x-y$, as the sum

$$
R_{0}(\omega, z)=\frac{1}{8 \pi^{3}} \int_{B_{\delta}} \frac{e^{-i \theta z}}{\phi(\theta)-\omega} d \theta+\frac{1}{8 \pi^{3}} \int_{T^{3} \backslash B_{\delta}} \frac{e^{-i \theta z}}{\phi(\theta)-\omega} d \theta=R_{01}(\omega, z)+R_{02}(\omega, z),
$$

where $B_{\delta}$ is the ball of radius $\delta$. Since $\phi(\theta)=|\theta|^{2}+\mathcal{O}\left(|\theta|^{4}\right)$, it follows that $R_{02}(\omega, z)$ is an analytic function of $\omega$ in $|\omega| \leq \delta^{2} / 2$, and

$$
\left|\partial_{\omega}^{j} R_{02}(\omega, z)\right| \leq \frac{C_{j, N}}{(|z|+1)}, \quad|\omega| \leq \delta^{2} / 2, \quad z \in \mathbb{Z}^{3} .
$$

Hence, it suffices to prove an asymptotics of type (2.10) for $R_{01}$. For simplicity, we suppose that $\phi(\theta)=|\theta|^{2}$. (If $\phi(\theta)=|\theta|^{2}+\mathcal{O}\left(|\theta|^{4}\right)$, the proof is similar, differing only in some technical details.) We choose a system of coordinates in which the direction of the axis $\theta_{3}$ coincides with the direction of the vector $z$, and we rewrite $R_{01}(\omega, z)$ as

$$
R_{01}(\omega, z)=\frac{1}{8 \pi^{3}} \int_{|n|=1}\left(\int_{0}^{\delta} \frac{e^{-i r|z| n_{3}} r^{2} d r}{r^{2}-\omega}\right) d S(n)
$$

Here $r=|\theta|, \theta=r n$. Then

$$
\begin{aligned}
R_{01}(\omega, z) & =\frac{1}{8 \pi^{3}} \int_{S_{+}}\left(\int_{0}^{\delta} \frac{e^{-i r|z| n_{3}} r^{2} d r}{r^{2}-\omega}+\int_{0}^{\delta} \frac{e^{i r|z| n_{3}} r^{2} d r}{r^{2}-\omega}\right) d S(n) \\
& =\frac{1}{8 \pi^{3}} \int_{S_{+}}\left(\int_{-\delta}^{\delta} \frac{e^{-i r|z| n_{3}} r^{2} d r}{r^{2}-\omega}\right) d S(n),
\end{aligned}
$$


where $S_{+}=\left\{|n|=1, n_{3}>0\right\}$. The integrand on the right-hand side of (2.11) has one simple pole in the lower half-plane. The Cauchy residue theorem yields

$$
\begin{aligned}
R_{01}(\omega, z) & =-\frac{i \sqrt{\omega}}{32 \pi^{4}} \int_{S_{1}^{+}} e^{i \sqrt{\omega}|z| n_{3}} d S(n)+\frac{1}{8 \pi^{3}} \int_{S_{+}}\left(\int_{\Gamma_{\delta}} \frac{e^{-i r|z| n_{3}} r^{2} d r}{r^{2}-\omega}\right) d S(n) \\
& =R_{01}^{1}(\omega, z)+R_{01}^{2}(\omega, z) .
\end{aligned}
$$

Here $\Gamma_{\delta}=\{|r|=\delta, \operatorname{Im} r<0\}$. For the first summand, an asymptotics of the type (2.10) is evident. For the second summand we have

$$
\begin{aligned}
R_{01}^{2}(\omega, z) & =\frac{1}{8 \pi^{3}} \int_{0}^{2 \pi} d \beta \int_{0}^{\pi / 2} d \alpha \int_{\Gamma_{\delta}} \frac{e^{-i r|z| \cos \alpha} r^{2} \sin \alpha d r}{r^{2}-\omega} \\
& =\frac{i}{4 \pi^{2}|z|} \int_{\Gamma_{\delta}} \frac{r d r}{r^{2}-\omega} \int_{0}^{\pi / 2} d e^{-i r|z| \cos \alpha} \\
& =\frac{i}{4 \pi^{2}|z|} \int_{\Gamma_{\delta}} \frac{r\left(1-e^{-i r|z|}\right) d r}{r^{2}-\omega}, \quad|z| \neq 0,
\end{aligned}
$$

where $\alpha$ is the angle between $z$ and $\theta$. Since $r^{2}=\delta^{2}$ on $\Gamma_{\delta}$, the operator-valued function $R_{01}^{2}(\omega)$ is analytic for $|\omega|<\delta^{2} / 2$. Moreover, the function $R_{01}^{2}(\omega)$ and all its derivatives with respect to $\omega$ are bounded in $\mathcal{B}(\sigma,-\sigma)$ for $\sigma>1 / 2$. Hence, $R_{01}^{2}$ admits an expansion of type (2.10).

Remark 2.5. The expansion (2.10) can be differentiated $N+1$ times in $\mathcal{B}(\sigma,-\sigma)$ with $\sigma>N+3 / 2$ :

$$
\partial_{\omega}^{r} R_{0}(\omega)=\partial_{\omega}^{r}\left(\sum_{k=0}^{N} A_{k} \omega^{k / 2}\right)+\mathcal{O}\left(\omega^{(N+1) / 2-r}\right), \quad 1 \leq r \leq N+1 .
$$

Proof. For the proof, we note that each differentiation of the resolvent with respect to $\omega$ increases the order of the pole of the integrand on the right in (2.11) by one. Therefore, the calculation of the corresponding residue leads to differentiation of the exponential, which results in an extra factor of $|z|$. Hence, $\sigma$ increases by one.

2.2.2. Hyperbolic points. Here we construct the Puiseux expansion of the free resolvent $R_{0}(\omega)$ near the "hyperbolic" point $\omega_{2}=4$ (the expansion near the point $\omega_{3}=8$ can be constructed similarly). The main contribution to (2.2) is given by the corresponding critical points $(0,0, \pi),(0, \pi, 0)$, and $(\pi, 0,0)$ of hyperbolic type.

Proposition 2.6. Let $N=-1,0,1, \ldots$, and let $\sigma>2 N+7 / 2$. Then in $\mathcal{B}(\sigma,-\sigma)$ we have

$$
R_{0}(4+\omega)=\sum_{k=0}^{N} E_{k} \omega^{k}+\sqrt{\omega} \sum_{k=0}^{N} B_{k} \omega^{k}+\mathcal{O}\left(\omega^{N+1}\right), \quad|\omega| \rightarrow 0, \quad \operatorname{Im} \omega>0 .
$$

Here the operators $E_{k}, B_{k}$ belong to $\mathcal{B}(\sigma,-\sigma)$ with $\sigma>2 k+3 / 2$. A similar expansion is valid for $\operatorname{Im} \omega<0$.

Proof. For $\omega=\omega_{2}=4$, the denominator of the integral (2.2) vanishes along the curve $\phi(\theta)=4$. We shall study the main contribution of the points $(0,0, \pi),(0, \pi, 0)$, and $(\pi, 0,0)$ of the surface, which are critical points of $\phi(\theta)$. The contribution of the other points of the curve can be computed by methods of Subsection 2.1. For definiteness, consider the integral over a neighborhood of the point $(\pi, 0,0)$. Let $\zeta(\theta)$ be a smooth 
cutoff function equal to 1 in a neighborhood of the point $(\pi, 0,0)$ (the other properties of $\zeta(\theta)$ will be specified below). For $\operatorname{Im} \omega>0$, denote

$$
\begin{aligned}
Q(\omega, z) & =\frac{1}{8 \pi^{3}} \int \frac{e^{-i z \theta} \zeta(\theta) d \theta}{\phi(\theta)-4-\omega} \\
& =\frac{e^{-i z_{3} \pi}}{8 \pi^{3}} \int \frac{e^{-i z \theta^{\prime}} \zeta_{1}\left(\theta^{\prime}\right) d \theta^{\prime}}{4 \sin ^{2} \frac{\theta_{1}}{2}+4 \sin ^{2} \frac{\theta_{2}}{2}-4 \sin ^{2} \frac{\theta_{3}^{\prime}}{2}-\omega},
\end{aligned}
$$

where $\theta_{3}^{\prime}=\theta_{3}-\pi, \theta^{\prime}=\left(\theta_{1}, \theta_{2}, \theta_{3}^{\prime}\right)$, and $\zeta_{1}\left(\theta^{\prime}\right)=\zeta(\theta)$. We assume that $\zeta_{1}\left(\theta^{\prime}\right)$ is symmetric in $\theta_{1}, \theta_{2}$ and $\theta_{3}^{\prime}$. Then the exponential in the numerator can be replaced by its even part, so that we have

$$
Q(\omega, z)=\frac{e^{-i z_{3} \pi}}{\pi^{3}} \int_{0}^{\infty} \int_{0}^{\infty} \int_{0}^{\infty} \frac{\cos \left(z_{1} \theta_{1}\right) \cos \left(z_{2} \theta_{2}\right) \cos \left(z_{3} \theta_{3}\right) \zeta_{1}(\theta) d \theta}{4 \sin ^{2} \frac{\theta_{1}}{2}+4 \sin ^{2} \frac{\theta_{2}}{2}-4 \sin ^{2} \frac{\theta_{3}}{2}-\omega}=\frac{e^{-i z_{3} \pi}}{\pi^{3}} Q_{1}(\omega, z) .
$$

We want to obtain an expansion of the form (2.13) for $Q_{1}$. We change the variables: $s_{i}=2 \sin \frac{\theta_{i}}{2}$, and choose a cutoff function $\zeta$ such that $\zeta_{1}(\theta)=\zeta_{2}\left(|s|^{2}\right)$, with a smooth function $\zeta_{2}$. Then

$$
\begin{aligned}
Q_{1}(\omega, z) & =\int_{0}^{\infty} \int_{0}^{\infty} \int_{0}^{\infty} \frac{F\left(z, s_{1}^{2}, s_{2}^{2}, s_{3}^{2}\right) \zeta_{2}\left(|s|^{2}\right) d s}{s_{1}^{2}+s_{2}^{2}-s_{3}^{2}-\omega}, \\
F\left(z, s_{1}^{2}, s_{2}^{2}, s_{3}^{2}\right) & =\prod_{i} \frac{2 \cos \left(2 z_{i} \arcsin s_{i} / 2\right)}{\sqrt{4-s_{i}^{2}}} .
\end{aligned}
$$

Now we use the cylindrical variables: $s_{1}=\tau \cos \varphi, s_{2}=\tau \sin \varphi, s_{3}=s_{3}$. Then

$$
\begin{aligned}
Q_{1}(\omega, z) & =\int_{0}^{\infty} \int_{0}^{\infty} \frac{F_{1}\left(z, \tau^{2}, s_{3}^{2}\right) \zeta_{2}\left(\tau^{2}+s_{3}^{2}\right) \tau d \tau d s_{3}}{\tau^{2}-s_{3}^{2}-\omega}, \\
F_{1}\left(z, \tau^{2}, s_{3}^{2}\right) & =\int_{0}^{\pi / 2} F\left(z, \tau^{2} \cos ^{2} \varphi, \tau^{2} \sin ^{2} \varphi, s_{3}^{2}\right) d \varphi .
\end{aligned}
$$

We change the variables once again:

$$
\rho_{1}=\tau^{2}-s_{3}^{2}=R^{2} \cos 2 \psi, \quad \rho_{2}=2 \tau s_{3}=R^{2} \sin 2 \psi,
$$

where $R, \psi$ are the polar coordinates on the plane $\left(\tau, s_{3}\right)$. Then $|\rho|^{2}=\rho_{1}^{2}+\rho_{2}^{2}=R^{4}$, whence $|\rho|=R^{2}, \tau^{2}=\left(|\rho|+\rho_{1}\right) / 2, s_{3}^{2}=\left(|\rho|-\rho_{1}\right) / 2, d \rho_{1} d \rho_{2}=4|\rho| d \tau d s_{3}$, and

$$
Q_{1}(\omega, z)=\int_{0}^{\infty}\left(\int_{\mathbb{R}} \frac{h\left(|\rho|, \rho_{1}, z\right)}{\left(\rho_{1}-\omega\right)|\rho|} \sqrt{|\rho|+\rho_{1}} d \rho_{1}\right) d \rho_{2},
$$

where $h\left(|\rho|, \rho_{1}, z\right)=F_{1}\left(z, \frac{|\rho|+\rho_{1}}{2}, \frac{|\rho|-\rho_{1}}{2}\right) \zeta_{2}(|\rho|) /(4 \sqrt{2})$. Now we can specify the final required property of our cutoff function:

$$
\operatorname{supp} \zeta_{2}(|\rho|) \cap\left\{\rho \in \mathbb{R}^{2}: \rho_{2} \geq 0\right\} \subset \Pi=\left\{\left(\rho_{1}, \rho_{2}\right):-\delta \leq \rho_{1} \leq \delta, 0 \leq \rho_{2} \leq \delta\right\}
$$

with some $0<\delta<1$.

Let $0<|\omega| \leq \delta / 2, \operatorname{Im} \omega>0$. Denote $r=|\rho|$. The function $h\left(r, \rho_{1}, z\right)$ can be expanded in the following finite Taylor series with respect to $\rho_{1}$ :

$$
h\left(r, \rho_{1}, z\right)=h_{0}(r, z)+h_{1}(r, z) \rho_{1}+\cdots+h_{N}(r, z) \rho_{1}^{N}+H_{N}\left(r, \rho_{1}, z\right) \rho_{1}^{N},
$$

where $h_{k}(r, z)$ is a polynomial in $z$ of order $2 k$, and

$$
\begin{aligned}
\left|H_{N}\left(r, \rho_{1}, z\right)\right| & \leq C|z|^{2 N} \\
\left|\partial_{\rho_{1}} H_{N}\left(r, \rho_{1}, z\right)\right| & \leq C|z|^{2 N+2}, \quad\left(\rho_{1}, \rho_{2}\right) \in[-\delta, \delta] \times[0, \delta] .
\end{aligned}
$$


We substitute (2.16) in (2.15). Then

$$
Q_{1}(\omega, z)=\sum_{k=0}^{N} J_{k}(\omega, z)+\widetilde{J}_{N}(\omega, z),
$$

where

$$
\begin{aligned}
J_{k}(\omega, z) & =\int_{\Pi} \frac{h_{k}(r, z) \rho_{1}^{k} \sqrt{r+\rho_{1}}}{\left(\rho_{1}-\omega\right) r} d \rho_{1} d \rho_{2}, \\
\widetilde{J}_{N}(\omega, z) & =\int_{\Pi} \frac{H_{N}\left(r, \rho_{1}, z\right) \rho_{1}^{N} d \rho_{1} d \rho_{2}}{\left(\rho_{1}-\omega\right) r} \sqrt{r+\rho_{1}} .
\end{aligned}
$$

Step i. First, we consider $J_{k}(\omega, z), k=0,1, \ldots, N$ :

$$
\begin{aligned}
J_{k}(\omega, z) & =\int_{\Pi} \frac{h_{k}(r, z) \sqrt{r+\rho_{1}}}{r}\left(\rho_{1}^{k-1}+\omega \rho_{1}^{k-2}+\cdots+\omega^{k-1}+\frac{\omega^{k}}{\rho_{1}-\omega}\right) d \rho_{1} d \rho_{2} \\
& =\sum_{k=0}^{k-1} a_{k, j}(z) \omega^{j}+\omega^{k} \int_{\Pi} \frac{h_{k}(r, z) \sqrt{r+\rho_{1}}}{\left(\rho_{1}-\omega\right) r} d \rho_{1} d \rho_{2} \\
& =\sum_{j=0}^{k-1} a_{k, j}(z) \omega^{j}+\omega^{k} \int_{0}^{\delta} \frac{h_{k}(r, z)}{\sqrt{r}} d r \int_{0}^{\pi} \frac{\sqrt{1+\cos \psi} d \psi}{\cos \psi-\omega / r}
\end{aligned}
$$

where the $a_{k, j}(z)$ are polynomials of order $2 k$. We calculate the integral

$$
\begin{aligned}
\int_{0}^{\pi} \frac{\sqrt{1+\cos \psi} d \psi}{\cos \psi-\omega / r} & =\int_{0}^{\pi} \frac{2 \sqrt{2} d\left(\sin \frac{\psi}{2}\right)}{1-2 \sin ^{2} \frac{\psi}{2}-\frac{\omega}{r}} \\
& =\int_{0}^{1} \frac{-\sqrt{2} d t}{t^{2}-\frac{r-\omega}{2 r}}=\frac{-\sqrt{r}}{\sqrt{r-\omega}} \log \frac{1-\sqrt{\frac{r-\omega}{2 r}}}{1+\sqrt{\frac{r-\omega}{2 r}}}+\frac{\pi i \sqrt{r}}{\sqrt{r-\omega}} .
\end{aligned}
$$

Here $\sqrt{r} \geq 0$, the function $z=\sqrt{r-\omega}$ is analytic for $\operatorname{Im} \omega>0$ with values in the domain $\operatorname{Im} z<0, \operatorname{Re} z>0$, and the function $\zeta=\log w$ is analytic for $|w|<1, \operatorname{Im} w>0$, where $\log (-1)=\pi i$.

Substituting (2.20) in (2.19), we get

$$
J_{k}(\omega, z)=\sum_{j=0}^{k-1} a_{k, j}(z) \omega^{j}+\omega^{k} \int_{0}^{\delta}\left(\pi i-\log \frac{1-\sqrt{\frac{r-\omega}{2 r}}}{1+\sqrt{\frac{r-\omega}{2 r}}}\right) \frac{h_{k}(r, z) d r}{\sqrt{r-\omega}} .
$$

We expand $h_{k}(r, z)$ in the following finite Taylor series with respect to $r$ :

$$
h_{k}(r, z)=h_{k, 0}(z)+h_{k, 1}(z) r+\cdots+h_{k, N-k}(z) r^{N-k}+H_{k, N-k}(r, z) r^{N-k},
$$

where the $h_{k, j}(z)$ are polynomials of order $2(k+j)$, and $\left|H_{k, N-k}(r, z)\right| \leq C|z|^{2 N}, 0 \leq$ $r \leq \delta$.

Lemma 2.7. If $0<|\omega|<\delta / 2, \operatorname{Im} \omega>0$, then

$$
I_{l}=\int_{0}^{\delta}\left(\pi i-\log \frac{1-\sqrt{\frac{r-\omega}{2 r}}}{1+\sqrt{\frac{r-\omega}{2 r}}}\right) \frac{r^{l} d r}{\sqrt{r-\omega}}=s_{l}(\omega)+C_{l} \omega^{l} \sqrt{\omega},
$$

where the $s_{l}$ are analytic in the domain $0<|\omega|<\delta / 2, \operatorname{Im} \omega>0$, and $C_{l} \in \mathbb{R}$. 
We shall prove this lemma in Appendix A. Now (2.21)-(2.23) imply that for $0<|\omega|<$ $\delta / 2, \operatorname{Im} \omega>0$ we have

$$
\begin{aligned}
J_{k}(\omega, z)= & \sum_{j=0}^{N} b_{k, j}(z) \omega^{j}+\omega^{k} \sqrt{\omega} \sum_{j=0}^{N-k} c_{k, j}(z) \omega^{j}+\widetilde{a}_{N, k}(\omega, z) \omega^{N+1} \\
& +\omega^{k} \int_{0}^{\delta}\left(\pi i-\log \frac{1-\sqrt{\frac{r-\omega}{2 r}}}{1+\sqrt{\frac{r-\omega}{2 r}}}\right) \frac{H_{k, N-k}(r, z) r^{N-k} d r}{\sqrt{r-\omega}},
\end{aligned}
$$

where $\left|b_{k, j}(z)\right| \leq C|z|^{2 N},\left|c_{k, j}(z)\right| \leq C|z|^{2(k+j)}$, and $\left|\widetilde{a}_{N, k}(\omega, z)\right| \leq C|z|^{2 N}$. Next,

$$
\int_{0}^{\delta}\left(\pi i-\log \frac{1-\sqrt{\frac{r-\omega}{2 r}}}{1+\sqrt{\frac{r-\omega}{2 r}}}\right) \frac{H_{k, N-k}(r, z) r^{N-k} d r}{\sqrt{r-\omega}}=\int_{0}^{2|\omega|}+\int_{2|\omega|}^{\delta}=\mathcal{I}_{1}+\mathcal{I}_{2} .
$$

In $\mathcal{I}_{1}$ we put $r=|\omega| \tau$, obtaining

$$
\begin{aligned}
\left|\mathcal{I}_{1}\right| & =|\omega|^{N-k} \sqrt{|\omega|}\left|\int_{0}^{2}\left(\pi i-\log \frac{1-\sqrt{\frac{\tau-\omega /|\omega|}{2 \tau}}}{1+\sqrt{\frac{\tau-\omega /|\omega|}{2 \tau}}}\right) \frac{H_{k, N-k}(|\omega| \tau, z) \tau^{N-k} d \tau}{\sqrt{\tau-\omega /|\omega|}}\right| \\
& \leq C|z|^{2 N}|\omega|^{N-k} \sqrt{|\omega|} .
\end{aligned}
$$

We expand $\sqrt{r-\omega}$ and the function in brackets in finite Taylor series with respect to $\omega / r$ :

$$
\begin{aligned}
\mathcal{I}_{2}= & \int_{2|\omega|}^{\delta} H_{k, N-k}(r, z) r^{N-k-1 / 2} \\
& \quad \times\left(d_{0}+d_{1} \frac{\omega}{r}+\cdots+d_{N-k} \frac{\omega^{N-k}}{r^{N-k}}+\widehat{d}_{N-k}(\omega / r) \frac{\omega^{N-k}}{r^{N-k}}\right) d r \\
= & \int_{2|\omega|}^{\delta} H_{k, N-k}(r, z) \\
& \quad \times\left(d_{0} r^{N-k-1 / 2}+d_{1} \omega r^{N-k-3 / 2}+\cdots+d_{N-k} \omega^{N-k} r^{-1 / 2}\right) d r+\widetilde{u}_{N-k}(\omega, z) \\
= & \int_{0}^{\delta} H_{k, N-k}(r, z) \\
& \quad \times\left(d_{0} r^{N-k-1 / 2}+d_{1} \omega r^{N-k-3 / 2}+\cdots+d_{N-k} \omega^{N-k} r^{-1 / 2}\right) d r+\widehat{u}_{N-k}(\omega, z) \\
= & \sum_{j=0}^{N-k} u_{j}(z) \omega^{j}+\widehat{u}_{N-k}(\omega, z),
\end{aligned}
$$

and we arrive at the estimates $\left|\widehat{d}_{N-k}(\omega / r)\right| \leq C,\left|u_{j}(z)\right| \leq C|z|^{2 N}$, as well as $\left|\widetilde{u}_{N-k}(\omega, z)\right|,\left|\widehat{u}_{N-k}(\omega, z)\right| \leq C|z|^{2 N}|\omega|^{N-k}$. Now (2.24)-(2.27) imply that

$$
J_{k}(\omega, z)=\sum_{j=0}^{N} d_{k, j}(z) \omega^{j}+\omega^{k} \sqrt{\omega} \sum_{j=0}^{N-k} c_{k, j}(z) \omega^{j}+\widetilde{d}_{N, k}(\omega, z),
$$

where $\left|d_{k, j}(z)\right| \leq C|z|^{2 N},\left|c_{k, j}(z)\right| \leq C|z|^{2(k+j)}$, and $\left|\widetilde{d}_{N, k}(\omega, z)\right| \leq C|z|^{2 N}|\omega|^{N}$. 
Step ii. It remains to consider the term $\widetilde{J}_{N}(\omega, z)$ on the right-hand side of (2.18):

$$
\begin{aligned}
\widetilde{J}_{N}(\omega, z)= & \int_{\Pi} \frac{H_{N}\left(r, \rho_{1}, z\right) \sqrt{r+\rho_{1}}}{r} \\
& \times\left(\rho_{1}^{N-1}+\omega \rho_{1}^{N-2}+\cdots+\omega^{N-1}+\frac{\omega^{N}}{\rho_{1}-\omega}\right) d \rho_{1} d \rho_{2} \\
= & \sum_{j=0}^{N-1} w_{j}(z) \omega^{j}+\omega^{N} \int_{\Pi} \frac{H_{N}\left(r, \rho_{1}, z\right) \sqrt{r+\rho_{1}} d \rho_{1} d \rho_{2}}{\left(\rho_{1}-\omega\right) r},
\end{aligned}
$$

where $\left|w_{j}(z)\right| \leq C|z|^{2 N}$.

Lemma 2.8. If $0<|\omega|<\delta / 2, \operatorname{Im} \omega>0$, then

$$
\left|\int_{\Pi} \frac{H_{N}\left(r, \rho_{1}, z\right) \sqrt{r+\rho_{1}} d \rho_{1} d \rho_{2}}{\left(\rho_{1}-\omega\right) r}\right| \leq C|z|^{2 N} \log ^{2}|z|, \quad|z|>1 .
$$

This lemma is proved in Appendix B.

Step iii. Finally, (2.18) and (2.28)-(2.30) imply that

$$
Q_{1}(\omega, z)=\sum_{k=0}^{N} q_{k}(z) \omega^{k}+\sqrt{\omega} \sum_{k=0}^{N} p_{k}(z) \omega^{k}+\widehat{q}_{N}(\omega, z), \quad|\omega| \rightarrow 0,
$$

where $\left|\widehat{q}_{N}(\omega, z)\right| \leq C|z|^{2 N} \log ^{2}|z||\omega|^{N}$. Next, we have $p_{k}(z)=\mathcal{O}\left(|z|^{2 k}\right)$ and $q_{k}(z)=$ $\mathcal{O}\left(|z|^{2 N}\right)$ for $0 \leq k \leq N$. Therefore, $q_{k}(z)=\mathcal{O}\left(|z|^{2 k}\right)$, because the $q_{k}(z)$ do not depend on $N$.

Corollary 2.9. Let $\sigma>3 / 2$. Then in $\mathcal{B}(\sigma,-\sigma)$ we have

$$
R_{0}(4+\omega)=\mathcal{O}(1), \quad|\omega| \rightarrow 0, \quad \operatorname{Im} \omega>0 .
$$

Corollary 2.10. The expansion (2.13) can be differentiated. More precisely,

$$
\partial_{\omega} R_{0}(4+\omega)=\frac{B_{0}}{2 \sqrt{\omega}}+\mathcal{O}(1), \quad|\omega| \rightarrow 0, \quad \operatorname{Im} \omega>0,
$$

in $\mathcal{B}(\sigma,-\sigma)$ with $\sigma>7 / 2$,

$$
\partial_{\omega}^{2} R_{0}(4+\omega)=-\frac{B_{0}}{4 \omega \sqrt{\omega}}+\mathcal{O}\left(\omega^{-1 / 2}\right), \quad|\omega| \rightarrow 0, \quad \operatorname{Im} \omega>0,
$$

in $\mathcal{B}(\sigma,-\sigma)$ with $\sigma>11 / 2$.

Proof. It suffices to obtain asymptotics of the form (2.32) and (2.33) for $Q_{1}$ (see (2.14)).

Formula (2.14) yields

$$
\begin{aligned}
\partial_{\omega} Q_{1} & (\omega, z)=\int_{0}^{\infty} \int_{0}^{\infty} \frac{F_{1}\left(z, \tau^{2}, s^{2}\right) \zeta_{2}\left(\tau^{2}+s^{2}\right) \tau d \tau d s}{\left(\tau^{2}-s^{2}-\omega\right)^{2}} \\
& =\int_{0}^{\infty} d s \int_{0}^{\infty} F_{1}\left(z, \tau^{2}, s^{2}\right) \zeta_{2}\left(\tau^{2}+s^{2}\right) \partial_{\tau} \frac{-1 / 2}{\tau^{2}-s^{2}-\omega} d \tau \\
& =-\frac{1}{2} \int_{0}^{\infty} \frac{F_{1}\left(z, 0, s^{2}\right) \zeta_{2}\left(s^{2}\right)}{s^{2}+\omega} d s+\int_{0}^{\infty} \int_{0}^{\infty} \frac{\partial_{\tau}^{2}\left(F_{1}\left(z, \tau^{2}, s^{2}\right) \zeta_{2}\left(\tau^{2}+s^{2}\right)\right) \tau}{\tau^{2}-s^{2}-\omega} d \tau d s \\
& =S_{1}(\omega, z)+S_{2}(\omega, z) .
\end{aligned}
$$

The asymptotics of $S_{2}(\omega, z)$ is similar to (2.31):

$$
S_{2}(\omega)=\mathcal{O}(1), \quad|\omega| \rightarrow 0, \quad \operatorname{Im} \omega>0,
$$


in $\mathcal{B}(\sigma,-\sigma)$ with $\sigma>7 / 2$. Observe that differentiation with respect to $\tau^{2}$ gives extra factors of $|z|^{2}$, so that the value of $\sigma$ increases by two in comparison with (2.31).

Consider $S_{1}(\omega, z)$ :

$$
S_{1}(\omega, z)=\int_{0}^{\delta} \frac{\left(F_{2}\left(z, s^{2}\right)-F_{2}(z,|\omega|)\right) d s}{s^{2}+\omega}+\int_{0}^{\delta} \frac{F_{2}(z,|\omega|) d s}{s^{2}+\omega} .
$$

For the function $F_{2}\left(z, s^{2}\right)=-F_{1}\left(z, 0, s^{2}\right) \zeta_{2}\left(s^{2}\right) / 2$ we have

$$
\left|F_{2}\left(z, s^{2}\right)\right| \leq C, \quad\left|\partial_{s^{2}} F_{2}\left(z, s^{2}\right)\right| \leq C|z|^{2}, \quad\left|\partial_{s^{2}}^{2} F_{2}\left(z, s^{2}\right)\right| \leq C|z|^{4} .
$$

We estimate the first integral on the right in (2.36) using the second bound in (2.37):

$$
\left|\int_{0}^{\delta} \frac{\left(F_{2}\left(z, s^{2}\right)-F_{2}(z,|\omega|)\right) d s}{s^{2}+\omega}\right| \leq C|z|^{2} \int_{0}^{\delta} \frac{\left|s^{2}-\right| \omega||}{\left|s^{2}+\omega\right|} d s \leq C|z|^{2} .
$$

For the second integral on the right in (2.36) we have

$$
\int_{0}^{\delta} \frac{F_{2}(z,|\omega|) d s}{s^{2}+\omega}=F_{2}(z,|\omega|) \frac{1}{2 \sqrt{-\omega}}\left(\log \frac{\delta-\sqrt{-\omega}}{\delta+\sqrt{-\omega}}-\pi i\right)=p(z) \frac{1}{\sqrt{\omega}}+q(\omega, z),
$$

where

$$
|p(z)|+|q(\omega, z)| \leq C, \quad 0<|\omega|<\delta / 2, \quad \operatorname{Im} \omega>0
$$

Therefore,

$$
S_{1}(\omega)=\frac{P_{1}}{\sqrt{\omega}}+\mathcal{O}(1), \quad|\omega| \rightarrow 0, \quad \operatorname{Im} \omega>0,
$$

in $\mathcal{B}(\sigma,-\sigma)$ with $\sigma>7 / 2$. Relations (2.35) and (2.39) imply the asymptotics of the first derivative. Next, we consider the second derivative:

$$
\begin{aligned}
\partial_{\omega}^{2} Q_{1} & (\omega, z)=\int_{0}^{\infty} \int_{0}^{\infty} \frac{2 F_{1}\left(z, \tau^{2}, s^{2}\right) \zeta_{2}\left(\tau^{2}+s^{2}\right) \tau d \tau d s}{\left(\tau^{2}-s^{2}-\omega\right)^{3}} \\
& =\int_{0}^{\infty} d s \int_{0}^{\infty} F_{1}\left(z, \tau^{2}, s^{2}\right) \zeta_{2}\left(\tau^{2}+s^{2}\right) \partial_{\tau} \frac{-1 / 2}{\left(\tau^{2}-s^{2}-\omega\right)^{2}} d \tau \\
& =\frac{1}{2} \int_{0}^{\infty} \frac{F_{1}\left(z, 0, s^{2}\right) \zeta_{2}\left(s^{2}\right)}{\left(s^{2}+\omega\right)^{2}} d s+\int_{0}^{\infty} \int_{0}^{\infty} \frac{\frac{\partial_{\tau^{2}}\left(F_{1}\left(z, \tau^{2}, s^{2}\right) \zeta_{2}\left(\tau^{2}+s^{2}\right)\right) \tau}{\left(\tau^{2}-s^{2}-\omega\right)^{2}}}{\quad} d \tau d s \\
& =U_{1}(\omega, z)+U_{2}(\omega, z) .
\end{aligned}
$$

The proof of the asymptotics for $U_{2}(\omega, z)$ is similar to that for the first derivative. We obtain

$$
U_{2}(\omega)=\frac{P_{2}}{\sqrt{\omega}}+\mathcal{O}(1), \quad|\omega| \rightarrow 0, \quad \operatorname{Im} \omega>0
$$

in $\mathcal{B}(\sigma,-\sigma)$ with $\sigma>11 / 2$. Now we consider $U_{1}(\omega, z)$ :

$$
\begin{aligned}
U_{1}(\omega, z)= & -\int_{0}^{\delta} \frac{F_{2}\left(z, s^{2}\right)}{\left(s^{2}+\omega\right)^{2}} d s \\
= & -\int_{0}^{\delta} \frac{F_{2}\left(z, s^{2}\right)-F_{2}(z,|\omega|)-F_{2}^{\prime}(z,|\omega|)\left(s^{2}-|\omega|\right)}{\left(s^{2}+\omega\right)^{2}} d s+\int_{0}^{\delta} \frac{\left.F_{2}(z,|\omega|)\right)}{\left(s^{2}+\omega\right)^{2}} d s \\
& +\int_{0}^{\delta} \frac{\left.F_{2}^{\prime}(z,|\omega|)\right)}{s^{2}+\omega} d s=U_{11}(\omega, z)+U_{12}(\omega, z)+U_{13}(\omega, z) .
\end{aligned}
$$

The last bound in (2.37) implies that

$$
\left.U_{11}(\omega, z)|\leq C| z\right|^{4} .
$$


As in (2.38), we see that

$$
\begin{gathered}
U_{13}(\omega, z)=\frac{p_{1}(z)}{\sqrt{\omega}}+q_{1}(\omega, z), \\
\left|p_{1}(z)\right|+\left|q_{1}(\omega, z)\right| \leq C|z|^{2}, \quad 0<|\omega|<\frac{\delta^{2}}{2}, \quad \operatorname{Im} \omega>0 .
\end{gathered}
$$

Finally,

$$
\begin{gathered}
U_{12}(\omega, z)=F_{2}(z,|\omega|)\left(\frac{1}{4 \omega \sqrt{-\omega}}\left(\pi i-\log \frac{1+\sqrt{-\omega} / \delta}{1-\sqrt{-\omega} / \delta}\right)+\frac{\delta}{2 \omega\left(\delta^{2}+\omega\right)}\right) \\
=F_{2}(z,|\omega|)\left(\frac{1}{4 \omega \sqrt{-\omega}}\left(\pi i-\frac{2 \sqrt{-\omega}}{\delta}+\frac{2 \omega \sqrt{-\omega}}{3 \delta^{3}}+\cdots\right)+\frac{1}{2 \omega \delta}\left(1-\frac{\omega}{\delta^{2}}+\cdots\right)\right) \\
=\frac{s_{2}(z)}{\omega \sqrt{\omega}}+\frac{p_{2}(z)}{\sqrt{\omega}}+q_{2}(\omega, z), \quad\left|s_{2}(z)\right|+\left|p_{2}(z)\right|+\left|q_{2}(\omega, z)\right| \leq C, \\
0<|\omega|<\frac{\delta^{2}}{2}, \quad \operatorname{Im} \omega>0 .
\end{gathered}
$$

Formulas (2.41)-(2.43) yield the asymptotics of the second derivative.

\section{§3. Perturbed Resolvent}

3.1. Limiting absorption principle. For the perturbed resolvent, the limiting absorption principle holds true.

Proposition 3.1. Suppose $V \in \mathcal{V}, \sigma>3 / 2$. Then the following limits as $\varepsilon \rightarrow 0+$ exist:

$$
R(\omega \pm i \varepsilon) \stackrel{B(\sigma,-\sigma)}{\longrightarrow} R(\omega \pm i 0), \quad \omega \in \Sigma \backslash\{0,4,8,12\} .
$$

Proof. Let $\omega \in \Sigma \backslash\{0,4,8,12\}$, and let $\sigma>3 / 2$. Since the potential $V$ has finite support, Proposition 2.2 shows that

$$
I+V R_{0}(\omega \pm i \varepsilon) \stackrel{\mathcal{B}(\sigma, \sigma)}{\longrightarrow} I+V R_{0}(\omega \pm i 0), \quad \varepsilon \rightarrow 0+.
$$

The kernel of the operator $I+V R_{0}(\omega \pm i 0)$ is trivial (see [13, Theorem 10]), and the operator $V R_{0}(\omega \pm i 0)$ is finite-dimensional. Consequently, the operator $I+V R_{0}(\omega \pm i 0)$ is invertible, and moreover,

$$
\left(I+V R_{0}(\omega \pm i \varepsilon)\right)^{-1} \stackrel{\mathcal{B}(\sigma, \sigma)}{\longrightarrow}\left(I+V R_{0}(\omega \pm i 0)\right)^{-1}, \quad \varepsilon \rightarrow 0+.
$$

Now, relations (2.4), (3.2) and the identity $R=R_{0}\left(I+V R_{0}\right)^{-1}$ imply the existence of the limits (3.1).

Remark 3.2. For $\omega \in \Sigma \backslash\{0,4,8,12\}$ the derivatives $\partial_{\omega}^{k} R(\omega \pm i 0)$ belong to $\mathcal{B}(\sigma,-\sigma)$ with $\sigma>3 / 2+k$.

Proof. This follows from Proposition [2.2 and the identity (see [6, Theorem 9.2])

$$
R^{(k)}=\left[(1-R V) R_{0}^{(k)}-\left(\begin{array}{c}
k \\
1
\end{array}\right) R^{\prime} V R_{0}^{k-1}-\cdots\right](1-V R), \quad k=1,2, \ldots .
$$

3.2. Asymptotics near critical points. Here we are going to obtain an asymptotic expansion for the perturbed resolvent $R(\omega)$ near the critical points $\omega_{k}, k=1,2,3,4$.

Definition 3.3. i) A set $\mathcal{W} \subset \mathcal{V}$ is said to be generic if for each $V \in \mathcal{V}$ we have $\alpha V \in \mathcal{W}$ for all $\alpha \in \mathbb{R}$ with the possible exception of a discrete set.

ii) We say that a property is fulfilled for "generic" $V$ if it is fulfilled for all $V$ in a generic subset $\mathcal{W} \subset \mathcal{V}$. 
Theorem 3.4. Let $\sigma>3 / 2$. Then for "generic" $V \in \mathcal{V}$ we have the following expansion:

$$
R(\omega)=D_{1}+\mathcal{O}(\sqrt{\omega}), \quad|\omega| \rightarrow 0, \quad \arg \omega \in(0,2 \pi),
$$

in the norm of $\mathcal{B}(\sigma,-\sigma)$.

Proof. We use the relation

$$
R(\omega)=T^{-1}(\omega) R_{0}(\omega), \quad \text { where } \quad T(\omega):=I+R_{0}(\omega) V .
$$

By (2.10),

$$
T(\omega)=I+A_{0} V+\mathcal{O}(\sqrt{\omega}), \quad|\omega| \rightarrow 0, \quad \arg \omega \in(0,2 \pi) .
$$

We prove that for "generic" $V \in \mathcal{V}$ the operator $T(\omega)$ is invertible in $l_{-\sigma}^{2}$ for all sufficiently small $|\omega|>0$. It suffices to check that the operator $T(0)=I+A_{0} V$ is invertible in $l_{-\sigma}^{2}$, or that the operator with the kernel

$$
\left(1+x^{2}\right)^{-\sigma / 2}\left(\delta(x-y)+A_{0} V(y)\right)\left(1+y^{2}\right)^{\sigma / 2}
$$

is invertible in $l^{2}$. Consider the operator

$$
\mathcal{A}(\alpha)=\operatorname{Op}\left[\left(1+x^{2}\right)^{-\sigma / 2}\left(\delta(x-y)+\alpha A_{0} V(y)\right)\left(1+y^{2}\right)^{\sigma / 2}\right]=1+\alpha \mathcal{K}, \quad \alpha \in \mathbb{C} .
$$

For $\sigma>3 / 2$,

$$
K(x, y)=\left(1+x^{2}\right)^{-\sigma / 2} A_{0} V(y)\left(1+y^{2}\right)^{\sigma / 2} \in l^{2}\left(\mathbb{Z}^{2} \times \mathbb{Z}^{2}\right) .
$$

Hence, $K(x, y)$ is a Hilbert-Schmidt kernel, so that the operator $\mathcal{K}=\operatorname{Op}(K(x, y))$ : $l^{2} \rightarrow l^{2}$ is compact. Further, $\mathcal{A}(\alpha)$ is analytic in $\alpha \in \mathbb{C}$, and $\mathcal{A}(0)$ is invertible. It follows that $\mathcal{A}(\alpha)$ is invertible for all $\alpha \in \mathbb{C}$ outside of a discrete set; see [1]. Thus, we can replace the original potential $V$ by $\alpha V$ with $\alpha$ arbitrarily close to 1, if necessary, to have $T(0)$ invertible.

Now (3.4) and (3.5) imply that

$$
R(\omega)=(I+T(0)+\mathcal{O}(\sqrt{\omega}))^{-1}\left(A_{0}+\mathcal{O}(\sqrt{\omega})\right)=T(0)^{-1} A_{0}+\mathcal{O}(\sqrt{\omega})
$$

for sufficiently small $|\omega|>0$.

Remark 3.5. i) The expansion of the resolvent near the second elliptic point $\omega_{4}=12$ is similar to (3.3).

ii) The expansion of the form (3.3) near the hyperbolic points $\omega_{2}=4$ and $\omega_{3}=8$ require larger values of $\sigma$. Namely, for "generic" $V \in \mathcal{V}$ we have

$$
R\left(\omega_{k}+\omega\right)=D_{k}+\mathcal{O}(\sqrt{\omega}), \quad|\omega| \rightarrow 0, \quad \operatorname{Im} \omega>0, \quad k=1,2,
$$

in the norm of $\mathcal{B}(\sigma,-\sigma)$ with $\sigma>7 / 2$.

iii) These expansions can be differentiated twice in $\mathcal{B}(\sigma,-\sigma)$ with $\sigma>5 / 2$ for elliptic points and with $\sigma>11 / 2$ for hyperbolic points. In these cases, $\partial_{\omega}^{2} R\left(\omega_{k}+\omega\right)=\mathcal{O}\left(\omega^{-3 / 2}\right)$, $k=1,2,3,4$.

\section{§4. LONG-TIME ASYMPTOTICS}

Now we apply Lemma 4.2 below, which is a version of [6, Lemma 10.2], to prove the following theorem.

Theorem 4.1. Let $\sigma>11 / 2$. Then for "generic" $V \in \mathcal{V}$ the following asymptotics is valid:

$$
\left\|e^{-i t H}-\sum_{j=1}^{n} e^{-i t \mu_{j}} P_{j}\right\|_{B(\sigma,-\sigma)}=\mathcal{O}\left(t^{-3 / 2}\right), \quad t \rightarrow \infty .
$$

Here $P_{j}$ denotes the projection to the eigenspace corresponding to the eigenvalue $\mu_{j} \in$ $\mathbb{R} \backslash[0,12], j=1, \ldots, n$. 
Proof. Estimate (4.1) is based on the formula

$$
e^{-i t H}=-\frac{1}{2 \pi i} \oint_{|\omega|=C} e^{-i t \omega} R(\omega) d \omega, \quad C>\max \left\{12 ;\left|\mu_{j}\right|, j=1, \ldots, n\right\} .
$$

The above integral is equal to the sum of the residues at the poles of $R(\omega)$ and the integral over the contour around the segment $[0,12]$, i.e.,

$$
e^{-i t H}-\sum_{j=1}^{n} e^{-i t \mu_{j}} P_{j}=\frac{1}{2 \pi i} \int_{[0,12]} e^{-i t \omega}(R(\omega+i 0)-R(\omega-i 0)) d \omega=\int_{[0,12]} e^{-i t \omega} P(\omega) d \omega .
$$

The main contribution to the long-time asymptotics is given by the integrals over neighborhoods of the critical points. For example, consider the integral over the neighborhood of the point $\omega_{1}=0$. The expansion (3.3) and Remark 3.5 imply that

$$
\partial^{k} P(\omega)=\mathcal{O}\left(\partial^{k} \sqrt{\omega}\right), \quad \omega \rightarrow+0, \quad \omega \in \mathbb{R}, \quad k=0,1,2,
$$

in $B(\sigma,-\sigma)$ with $\sigma>11 / 2$. The following result is a special case of [6, Lemma 10.2].

Lemma 4.2. Assume $\mathcal{B}$ is a Banach space, $a>0$, and $F \in C(0, a ; \mathcal{B})$ satisfies $F(0)=$ $F(a)=0, F^{\prime} \in L^{1}(0, a ; \mathcal{B})$, and $F^{\prime \prime}(\omega)=\mathcal{O}\left(\omega^{-3 / 2}\right)$ as $\omega \rightarrow 0$. Then

$$
\int_{[0, a]} e^{-i t \omega} F(\omega) d \omega=\mathcal{O}\left(t^{-3 / 2}\right), \quad t \rightarrow \infty .
$$

Set $F(\omega)=\zeta(\omega) P(\omega)$, where $\zeta$ is a smooth function such that $\zeta(\omega)=1$ for $\omega \epsilon$ $[-1 / 2,1 / 2]$ and $\operatorname{supp} \zeta \subset(-1,1)$, and let $a=1$ and $\mathcal{B}=B(\sigma,-\sigma)$ with $\sigma>11 / 2$. Then estimate (4.3) allows us to apply Lemma 4.2, obtaining

$$
\int_{[0,1]} e^{-i t \omega} \zeta(\omega) P(\omega)=\mathcal{O}\left(t^{-3 / 2}\right), \quad t \rightarrow \infty,
$$

in $B(\sigma,-\sigma)$ with $\sigma>11 / 2$.

The integrals over neighborhoods of the other critical points can be treated similarly.

\section{§5. KLEIN-GORdON EQUATION}

Now we extend the results of $\S \$ 34$ to the case of the Klein-Gordon equation (1.4). Denote $\boldsymbol{\Psi}(t) \equiv(\psi(\cdot, t), \dot{\psi}(\cdot, t)), \mathbf{\Psi}_{0} \equiv\left(\psi_{0}, \pi_{0}\right)$. Then (1.4) becomes

$$
i \dot{\boldsymbol{\Psi}}(t)=\mathbf{H} \Psi(t)=\left(\begin{array}{cc}
0 & i \\
i\left(\Delta-m^{2}-V\right) & 0
\end{array}\right) \boldsymbol{\Psi}(t), \quad t \in \mathbb{R} ; \quad \boldsymbol{\Psi}(0)=\mathbf{\Psi}_{0} .
$$

The resolvent $\mathbf{R}(\omega)$ can be expressed in terms of the resolvent $R(\omega)$ as

$$
\mathbf{R}(\omega)=\left(\begin{array}{cc}
\omega R\left(\omega^{2}-m^{2}\right) & i R\left(\omega^{2}-m^{2}\right) \\
-i\left(1+\omega^{2} R\left(\omega^{2}-m^{2}\right)\right) & \omega R\left(\omega^{2}-m^{2}\right)
\end{array}\right), \quad \omega^{2}-m^{2} \in \mathbb{C} \backslash[0,12] .
$$

The representation (5.1) and the properties of $R(\omega)$ imply the following long-time asymptotics.

Suppose $\sigma>11 / 2$ and $\boldsymbol{\Psi}_{0} \in l_{\sigma}^{2} \oplus l_{\sigma}^{2}$. Then for "generic" $V \in \mathcal{V}$ we have

$$
\left\|e^{-i t \mathbf{H}} \mathbf{\Psi}_{0}-\sum_{ \pm} \sum_{j=1}^{n} e^{-i t \nu_{j}^{ \pm}} \mathbf{P}_{j}^{ \pm} \mathbf{\Psi}_{0}\right\|_{l_{-\sigma}^{2} \oplus l_{-\sigma}^{2}}=\mathcal{O}\left(t^{-3 / 2}\right), \quad t \rightarrow \infty .
$$

Here the $\mathbf{P}_{j}^{ \pm}$are the projections onto the eigenspaces corresponding to the eigenvalues $\nu_{j}^{ \pm}= \pm \sqrt{m^{2}+\mu_{j}}, j=1, \ldots, n$. 


\section{§6. Appendix A}

Lemma 2.7 will be proved by induction. For $l=0$ we get

$$
\begin{aligned}
I_{0} & =\int_{0}^{\delta}\left(\pi i-\log \frac{1-\sqrt{\frac{r-\omega}{2 r}}}{1+\sqrt{\frac{r-\omega}{2 r}}}\right) \frac{d r}{\sqrt{r-\omega}} \\
& =\left.2 \sqrt{r-\omega}\left(\pi i-\log \frac{1-\sqrt{\frac{r-\omega}{2 r}}}{1+\sqrt{\frac{r-\omega}{2 r}}}\right)\right|_{0} ^{\delta}+2 \sqrt{2} \omega \int_{0}^{\delta} \frac{d r}{\sqrt{r}(r+\omega)} \\
& =2 \sqrt{\delta-\omega}\left(\pi i-\log \frac{1-\sqrt{\frac{\delta-\omega}{2 \delta}}}{1+\sqrt{\frac{\delta-\omega}{2 \delta}}}\right)-\left.i 2 \sqrt{2 \omega} \log \frac{\sqrt{r}-i \sqrt{\omega}}{\sqrt{r}+i \sqrt{\omega}}\right|_{0} ^{\delta} \\
& =\widetilde{s}_{0}(\omega)-i 2 \sqrt{2 \omega} \log \frac{1-i \sqrt{\frac{\omega}{\delta}}}{1+i \sqrt{\frac{\omega}{\delta}}}-\pi \sqrt{2 \omega}=s_{0}(\omega)+C_{0} \sqrt{\omega},
\end{aligned}
$$

where $\widetilde{s}_{0}$ and $s_{0}$ are analytic functions of $\omega, C_{0}=-\pi \sqrt{2}$.

Next, for $l \geq 1$ we have

$$
\begin{aligned}
I_{l}= & \int_{0}^{\delta}\left(\pi i-\log \frac{1-\sqrt{\frac{r-\omega}{2 r}}}{1+\sqrt{\frac{r-\omega}{2 r}}}\right) \frac{r^{l} d r}{\sqrt{r-\omega}}=2 \delta^{l} \sqrt{\delta-\omega}\left(\pi i-\log \frac{1-\sqrt{\frac{\delta-\omega}{2 \delta}}}{1+\sqrt{\frac{\delta-\omega}{2 \delta}}}\right) \\
& -2 l \int_{0}^{\delta} \frac{r^{l-1}(r-\omega)}{\sqrt{r-\omega}}\left(\pi i-\log \frac{1-\sqrt{\frac{r-\omega}{2 r}}}{1+\sqrt{\frac{r-\omega}{2 r}}}\right) d r+2 \sqrt{2} \omega \int_{0}^{\delta} \frac{r^{l} d r}{\sqrt{r}(r+\omega)} \\
= & \widetilde{s}_{l}(\omega)-2 l I_{l}+2 l \omega I_{l-1}+2 \sqrt{2} \omega \int_{0}^{\delta} \frac{d r}{\sqrt{r}}\left(r^{l-1}-\omega r^{l-2}+\cdots+(-\omega)^{l-1}+\frac{(-\omega)^{l}}{r+\omega}\right) \\
= & \widetilde{s}_{l}(\omega)-2 l I_{l}+2 l \omega I_{l-1}+\widetilde{\widetilde{s}}_{l}(\omega)-i 2 \sqrt{2 \omega}(-\omega)^{l} \log \frac{1-i \sqrt{\frac{\omega}{\delta}}}{1+i \sqrt{\frac{\omega}{\delta}}}-2 \pi \sqrt{2 \omega}(-\omega)^{l},
\end{aligned}
$$

where $\widetilde{s}_{l}$ and $\widetilde{\widetilde{s}}_{l}$ are analytic functions of $\omega$. Consequently, $I_{l}=s_{l}(\omega)+C_{l} \omega^{l} \sqrt{\omega}$, where the $s_{l}$ are analytic functions of $\omega$ and $C_{l} \in \mathbb{R}$.

\section{$\S 7$. Appendix B}

Here we prove Lemma 2.8. We estimate only the integral over $\Pi_{+}=\left\{0 \leq \rho_{1}, \rho_{2} \leq \delta\right\}$. The integral over $\Pi \backslash \Pi_{+}$can be estimated similarly. We split the integral over $\Pi_{+}$into two integrals:

$$
\begin{aligned}
\int_{\Pi_{+}} \frac{H_{N}\left(r, \rho_{1}, z\right) \sqrt{r+\rho_{1}} d \rho_{1} d \rho_{2}}{\left(\rho_{1}-\omega\right) r}= & \int_{\Pi_{+}} \frac{\left(H_{N}\left(r, \rho_{1}, z\right)-H_{N}(r,|\omega|, z)\right) \sqrt{r+\rho_{1}} d \rho_{1} d \rho_{2}}{\left(\rho_{1}-\omega\right) r} \\
& +\int_{\Pi_{+}} \frac{H_{N}(r,|\omega|, z) \sqrt{r+\rho_{1}} d \rho_{1} d \rho_{2}}{\left(\rho_{1}-\omega\right) r}=J_{1}+J_{2} .
\end{aligned}
$$

As in (2.20), we obtain

$$
J_{2}=\int_{0}^{\delta}\left(\pi i-\log \frac{1-\sqrt{\frac{r-\omega}{2 r}}}{1+\sqrt{\frac{r-\omega}{2 r}}}\right) \frac{H_{N}(r,|\omega|, z) d r}{\sqrt{2(r-\omega)}} .
$$


Note that

$$
\begin{aligned}
|\log | \frac{\sqrt{2 r}-\sqrt{r-\omega}}{\sqrt{2 r}+\sqrt{r-\omega}}|| & =|\log | \frac{r+\omega}{(\sqrt{2 r}+\sqrt{r-\omega})^{2}}|| \\
& \leq|\log | r+\omega||+2|\log | \sqrt{2 r}+\sqrt{r-\omega}|| \leq 2|\log | r-|\omega||| .
\end{aligned}
$$

Then (2.8) implies that

$$
\left|J_{2}\right| \leq C|z|^{2 N} \int_{0}^{\delta} \frac{1+|\log | r-|\omega|||}{|\sqrt{r-|\omega|}|} d r \leq C|z|^{2 N} .
$$

Next, for $|z|>1$ we split $J_{1}$ as follows:

$$
J_{1}=J_{11}+J_{12}+J_{13},
$$

where $J_{1 j}$ is an integral over $\Pi_{j}, j=1,2,3$, with

$$
\begin{aligned}
& \Pi_{1}=\left\{\left(\rho_{1}, \rho_{2}\right) \in \Pi_{+}:|r|<1 /|z|^{4 / 3}\right\}, \\
& \Pi_{2}=\left\{\left(\rho_{1}, \rho_{2}\right) \in \Pi_{+} \backslash \Pi_{1}:\left|\rho_{1}-\right| \omega||<1 /|z|^{8 / 3}\right\}, \\
& \Pi_{3}=\Pi_{+} \backslash\left(\Pi_{1} \cup \Pi_{2}\right)
\end{aligned}
$$

(see Figure 1).

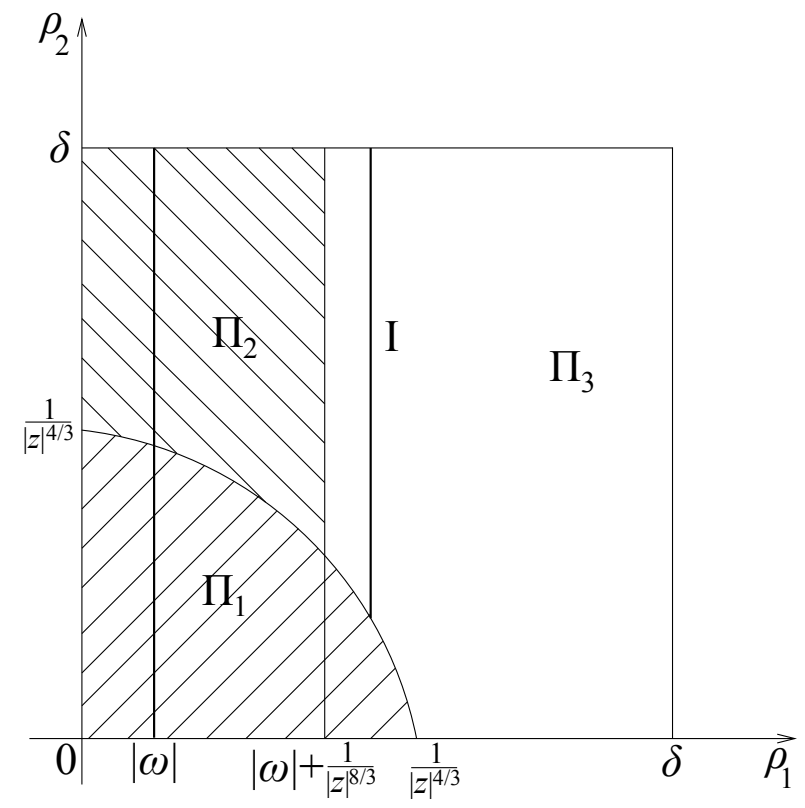

Figure 1. Case where $|\omega|-1 /|z|^{8 / 3}<0$.

Using (2.17) and the inequality $\left|\rho_{1}-\omega\right| \geq\left|\rho_{1}-\right| \omega||$, we get

$$
\begin{aligned}
\left|J_{11}\right| & \leq C|z|^{2 N+2} \int_{\Pi_{1}} \frac{\left|\rho_{1}-\right| \omega|| \sqrt{r+\rho_{1}} d \rho_{1} d \rho_{2}}{\left|\rho_{1}-\omega\right| r} \leq C|z|^{2 N+2} \int_{\Pi_{1}} \frac{\sqrt{r+\rho_{1}} d \rho_{1} d \rho_{2}}{r} \\
& \leq C|z|^{2 N+2} \int_{0}^{\pi / 2} d \psi \int_{0}^{1 /|z|^{4 / 3}} \sqrt{r+r \cos \psi} d r \\
& \leq C|z|^{2 N+2} \int_{0}^{\pi / 2} \cos \frac{\psi}{2} d \psi \int_{0}^{1 /|z|^{4 / 3}} \sqrt{r} d r \leq C|z|^{2 N} .
\end{aligned}
$$


Similarly, for the second integral we obtain

$$
\begin{aligned}
\left|J_{12}\right| & \leq C|z|^{2 N+2} \int_{\Pi_{2}} \frac{\sqrt{r+\rho_{1}} d \rho_{1} d \rho_{2}}{r} \\
& \leq C|z|^{2 N+2} \int_{\Pi_{2}} \frac{d \rho_{1} d \rho_{2}}{\sqrt{r}} \leq C|z|^{2 N+2}|z|^{2 / 3} \frac{\delta}{|z|^{8 / 3}} \leq C|z|^{2 N},
\end{aligned}
$$

because $1 / \sqrt{r} \leq|z|^{2 / 3}$ for $\left(\rho_{1}, \rho_{2}\right) \in \Pi_{2}$, and $\left|\Pi_{2}\right| \leq 2 \delta /|z|^{8 / 3}$. Finally, (2.17) implies that

$$
\left|J_{13}\right| \leq C|z|^{2 N} \int_{\Pi_{3}} \frac{d \rho_{1} d \rho_{2}}{\left|\rho_{1}-\right| \omega|| \sqrt{\rho_{1}^{2}+\rho_{2}^{2}}} \leq C|z|^{2 N} \log ^{2}|z|,
$$

because for any vertical interval $I \in \Pi_{3}$ we have

$$
\int_{I} \frac{d \rho_{2}}{\sqrt{\rho_{1}^{2}+\rho_{2}^{2}}}=\log \left(\rho_{2}+\sqrt{\rho_{1}^{2}+\rho_{2}^{2}}\right) \leq C \log |z|
$$

\section{§8. Appendix C. Asymptotic completeness}

We apply the results obtained above to construct the asymptotic scattering states. Let $u_{k}$ be eigenfunctions of the operator $H$ corresponding to the eigenvalues $\mu_{k}$, and let $U_{0}(t)$ be the dynamical group of the free Schrödinger equation.

Theorem 8.1. Suppose $\sigma>11 / 2$ and $\psi_{0} \in l_{\sigma}^{2}$. Then, for "generic" $V \in \mathcal{V}$, the solution of (1.1) admits the following long-time asymptotics:

$$
\psi(\cdot, t)=\sum_{k=1}^{n} C_{k} e^{-i t \mu_{k}} u_{k}+U_{0}(t) \phi_{ \pm}+r_{ \pm}(t), \quad t \rightarrow \pm \infty,
$$

where the $\phi_{ \pm} \in l^{2}$ are the corresponding scattering states, and

$$
\left\|r_{ \pm}(t)\right\|_{l^{2}}=\mathcal{O}\left(|t|^{-1 / 2}\right) .
$$

Proof. For definiteness, we consider the case where $t \rightarrow+\infty$. We apply the projection $P^{c}$ onto the continuous spectrum of the operator $H$ to both sides of (1.1):

$$
i P^{c} \dot{\psi}=P^{c} H \psi=H_{0} P^{c} \psi+V P^{c} \psi,
$$

because $P^{c}$ and $H$ commute. Applying the Duhamel representation to equation (8.2), we obtain

$$
P^{c} \psi(t)=U_{0}(t) P^{c} \psi(0)+\int_{0}^{t} U_{0}(t-\tau) V P^{c} \psi(\tau) d \tau, \quad t \in \mathbb{R} .
$$

This formula can be rewritten as

$$
\begin{aligned}
P^{c} \psi(t) & =U_{0}(t)\left(P^{c} \psi(0)+\int_{0}^{\infty} U_{0}(-\tau) V P^{c} \psi(\tau) d \tau\right)-\int_{t}^{\infty} U_{0}(t-\tau) V P^{c} \psi(\tau) d \tau \\
& =U_{0}(t) \phi+r_{+}(t) .
\end{aligned}
$$

We show that the integrals converge, and the function

$$
\phi_{+}=P^{c} \psi(0)+\int_{0}^{\infty} U_{0}(-\tau) V P^{c} \psi(\tau) d \tau
$$

belongs to $l^{2}$. Indeed, (4.1) implies that

$$
\begin{aligned}
\int_{0}^{\infty}\left\|U_{0}(-\tau) V P^{c} \psi(\tau)\right\|_{l^{2}} d \tau & =\int_{0}^{\infty}\left\|V P^{c} \psi(\tau)\right\|_{l^{2}} d \tau \leq C \int_{0}^{\infty}\left\|P^{c} \psi(\tau)\right\|_{l_{-\sigma}^{2}} d \tau \\
& \leq C \int_{0}^{\infty} t^{-3 / 2}\|\psi(0)\|_{l_{\sigma}^{2}} d \tau \leq C
\end{aligned}
$$


Here we have used the fact that $U_{0}(t)$ is unitary in $l^{2}$ and the identity $P^{c}=I-P^{d}$, where $P^{d}$ is the projection onto the subspace corresponding to the discrete spectrum, which consists of functions that decay exponentially. The estimate for $r_{+}(t)$ is proved similarly.

For the Klein-Gordon equation, an asymptotics of the form (8.1) is also valid.

\section{REFERENCES}

[1] P. M. Blekher, Operators that depend meromorphically on a parameter, Vestnik Moskov. Univ. Ser. I Mat. Mekh. 1969, no. 5, 30-36. (Russian) MR0265956 (42:865)

[2] B. R. Vaĭnberg, The short-wave asymptotic behavior of the solutions of stationary problems, and the asymptotic behavior as $t \rightarrow \infty$ of the solutions of nonstationary problems, Uspekhi Mat. Nauk 30 (1975), no. 2, 3-55; English transl., Russian Math. Surveys 30 (1975), no. 2, 1-58. MR0415085 $(54: 3176)$

[3] - Asymptotic methods in equations of mathematical physics, Moskov. Gos. Univ., Moscow, 1982; English transl., Gordon and Breach Sci. Publ., New York, 1989. MR.0700559 (85k:35033) MR 1054376 (91h:35081)

[4] I. M. Glazman, Direct methods of the qualitative spectral analysis of singular differential operators, Fizmatgiz, Moscow, 1963; English transl., Israel Program for Sci. Transl., Jerusalem, 1965; Daniel Davey and Co., Inc., New York, 1966. MR0185471 (32:2938) MR0190800 (32:8210)

[5] H. Islami and B. Vainberg, Large time behavior of solutions to difference wave operators, Comm. Partial Differential Equations 31 (2006), no. 1-3, 397-416. MR2209760 (2006j:39017)

[6] A. Jensen and T. Kato, Spectral properties of Schrödinger operators and time-decay of the wave functions, Duke Math. J. 46 (1979), 583-611. MR0544248(81b:35079)

[7] A. Jensen and G. Nenciu, A unified approach to resolvent expansions at thresholds, Rev. Math. Phys. 13 (2001), no. 6, 717-754. MR1841744 (2002e:81031)

[8] A. Komech, E. Kopylova, and M. Kunze, Dispersive estimates for $1 D$ discrete Schrödinger and Klein-Gordon equations, J. Appl. Anal. 85 (2006), no. 12, 1487-1508. MR.2282998 (2007i:35194)

[9] A. Komech, E. Kopylova, and B. Vainberg, On dispersive properties of discrete $2 D$ Schrödinger and Klein-Gordon equations, J. Funct. Anal. 254 (2008), 2227-2254. MR2402107 (2009f:35279)

[10] P. D. Lax and R. S. Phillips, Scattering theory, 2nd ed., Pure Appl. Math., vol. 26, Acad. Press, Inc., Boston, MA, 1989. MR1037774 (90k:35005)

[11] M. Murata, Asymptotic expansions in time for solutions of Schrödinger-type equations, J. Funct. Anal. 49 (1982), 10-56. MR0680855 (85d:35019)

[12] W. Schlag, Dispersive estimates for Schrödinger operators: A survey, Mathematical Aspects of Nonlinear Dispersive Equations, Ann. of Math. Stud., vol. 163, Princeton Univ. Press, Princeton, NJ, 2007, pp. 255-285; Preprint math.AP/0501037. MR.2333215 (2009k:35043)

[13] W. Shaban and B. Vainberg, Radiation conditions for the difference Schrödinger operators, J. Appl. Anal. 80 (2001), no. 3-4, 525-556. MR1914696 (2003d:47049)

[14] M. S. Èskina, The scattering problem for partial-difference equations, Mat. Fiz., No. 3, Naukova Dumka, Kiev, 1967, pp. 248-273. (Russian) MR0211027 (35:1912)

Institute for Information Transmission Problems, Russian Academy of Sciences, B. KaretNYi 19, Moscow 101447, Russia

E-mail address: ek@vpti.vladimir.ru

Received 19/NOV/2009

Translated by THE AUTHOR 